\title{
EPR-Untersuchungen an Radikalen und Triplettzuständen von Fluorenderivaten
}

\author{
Reinhard Stösser, Jörn-Uwe Thurner, Georg Tomaschewski \\ Humboldt-Universität zu Berlin, Sektion Chemie, Berlin \\ Uwe Ewert, Peter Heblik, Wilfried Schneider \\ AdW der DDR, Zentrum für wissenschaftlichen Gerätebau, Berlin* \\ Thomas Hanke \\ AdW der DDR, Zentralinstitut für Molekularbiologie, Berlin** \\ Z. Naturforsch. 37 a, 1241-1246 (1982); eingegangen am 10. Juni 1982 \\ EPR-Investigations on Radicals and Triplet-States of Fluorene-Derivatives \\ The photolysis of 9-diazolluorene-derivatives yields various paramagnetic species. By EPR- \\ spectıoscopy at continuous and modulated optical excitation these species have been identified as \\ radicals as well as triplets in ground- and excited states. In the simulation and interpretation of \\ the spectra comparison with structure analogous compounds and products was helpfull. On the \\ basis of the results the possible role of the paramagnetic intermediates in the reaction-scheme is \\ discussed.
}

\section{Einleitung}

Innerhalb der aromatischen Verbindungen ist die Fluorenstruktur von besonderem Interesse, da eine relativ starre Anordnung vorliegt, bei der Substituenten bzw. spezielle elektronische Effekte nicht nur an aromatischen Teilsystemen, sondern auch an einem $\mathbf{s p}^{3}$-hybridisierten Kohlenstoffatom lokalisiert werden können. Prinzipiell lassen sich Fluoren und seine Derivate als $2.2^{\prime}$-verbrückte Diphenyle auffassen, wobei die Verbrückung auch über verschiedene Atome bzw. Atomgruppen erfolgen kann. Damit ergeben sich nicht nur Analogien zum Biphenyl, sondern auch zum Dibenzofuran, Carbazol, Dibenzothiophen, Phenanthren und schließlich zu Triarylaminen und ähnlichen. Über Grundzustandseigenschaften dieser Verbindungen liegen zahlreiche Untersuchungen vor $[1-5]$.

Fluoren und seine Derivate bieten auch die Möglichkeit, Bildung und Reaktionen von Carbenen, insbesondere der entsprechenden Fluorenylidene, im Bereich $T \geqq 77 \mathrm{~K}$ spektroskopisch zu untersuchen. Die Beteiligung von Fluoren und seinen Derivaten an photochemischen Reaktionen kann unter geeigne-

\footnotetext{
* DDR-1199 Berlin, Rudower Chaussee 6.

** DDR-1150 Berlin, Lindenberger Weg 70.
}

Sonderdruckanforderungen an Doz. Dr. R. Stößer, Humboldt-Universität zu Berlin, Sektion Chemie, Hessische Str. 1-2, DDR-1040 Berlin. ten Bedingungen zu radikalischen Spezies sowie Triplettzuständen führen.

Ausgangspunkt für die hier dargestellten Untersuchungen war die Photolyse von 9-Diazofluoren in Pyridin, die bei Raumtemperatur zur Bildung einer tief blau gefärbten Verbindung $\left(\lambda_{\max }: 598 \mathrm{~nm}\right)$ führt, die als 1-(9-Fluorenyl-) pyridinium-Ylid identifiziert werden konnte $[6,7]$ (s.a. [2]).

Die entsprechende thermische Reaktion liefert demgegenüber als wesentliches Produkt das Fluorenonazin [7].

Nach bisher vorliegenden Befunden sollte der Mechanismus beider Reaktionen durch intermediär auftretende Carbene und radikalische Spezies wesentlich bestimmt werden. Ausgehend von EPR- und optischen Untersuchungen an paramagnetischen Fluorenspezies $[5,8]$ sollten deshalb die bei den obengenannten Reaktionen beobachteten Intermediate identifiziert und ein Beitrag zur Klärung ihrer Rolle im Mechanismus erbracht werden. Der Continuous Wave (CW)-EPR kommt hier neben der zweifellos vorteilhaften Optical Detection of Magnetic Resonance (ODMR) Bedeutung zu, da bisher nicht geklärt ist, ob die bei der stationären optischen Anregung ebenfalls angeregten carbenischen und radikalischen Zwischenstufen selbst eine ausreichende Emission zeigen. Wertvolle Informationen lassen sich natürlich auch anhand von Chemically Induced Dynamic Nuclear Polarisation (CIDNP)-Untersu- 


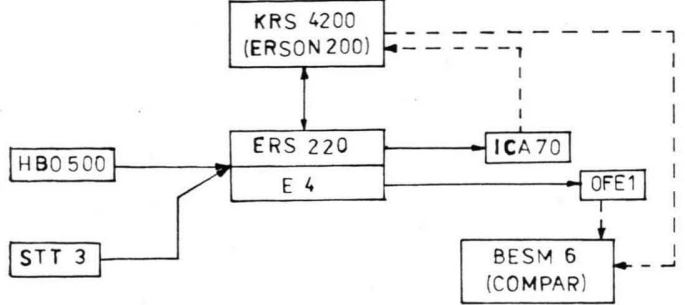

Abb. 1. Schematische Darstellung der verwendeten Hilfsmittel.

chungen an diesen Systemen erhalten (s. z. B. [9]). Bisher liegen verschiedene Untersuchungen zu Radikalen und Triplettzuständen von Fluorenderivaten in unterschiedlichen Medien einschließlich X-Traps in Festkörpern vor (s. z. B. [10]). In der vorliegenden Arbeit sollten folgende Aspekte berücksichtigt werden:

Vergleichende Untersuchungen an analogen optisch angeregten Verbindungen sowie isolierten Reaktionsprodukten (z. B. 9.9'-Bifluorenyl, Bifluorenyliden, Fluoren und Fluorenylderivaten) zur Interpretation von EPR-Spektren bestrahlter DiazofluorenDerivate in verschiedenen Matrizen (s. a. [11]).

Spektrensimulation auf der Basis eines phänomenologischen Spin-Hamilton-Operators zur einheitlichen Bestimmung der Nullfeldparameter. Diese Simulation und insbesondere die dabei ermittelte Winkelabhängigkeit der Resonanzwerte der magnetischen Induktion $\boldsymbol{B}$ und der Übergangswahrscheinlichkeiten für Triplettzustände mit Nullfeldaufspaltungen kleiner bzw. größer als die Energie der EPRMikrowellenquanten (X-Band), sollte dazu beitragen, den Habitus der Spektren ungeordneter Proben $\mathrm{zu}$ verstehen und Voraussetzungen für eine unverfälschte Registrierung der Signale zu schaffen.

Durchführung kinetischer Untersuchungen mit dem Ziel, Informationen über die Geschwindigkeit radikalischer Reaktionen der Carbene in flüssiger Phase zu gewinnen und die CW-EPR- und optischen Tieftemperatur-Untersuchungen zu ergänzen.

\section{Experimentelles}

Das Schema der verwendeten Gerätetechnik zeigt Abbildung 2. Als EPR-Spektrometer kamen die Typen ERS 220 (Hersteller: ZWG der AdW der DDR) und E 4 (Hersteller: Fa. Varian, USA) zur Anwendung. Die optische Anregung der Proben erfolgte mit Hilfe einer 500-W-Hg-Höchstdrucklampe (HBO 500, VEB Narva, DDR) stationär bzw. periodisch unter Verwendung eines mechanischen Choppers. Zur Selektion des Anregungslichtes wurden Wasserfilter und nachgeschaltete Farbgläser (VEB-Jenaer Glaswerke, Schott u. Gen.) verwendet.

Bei periodischer Anregung wurde der Signalnachweis über einen Vielkanalanalysator ICA 70 (Hersteller KFKI, VR Ungarn) geführt. Zur Temperierung der Proben diente wahlweise ein $\mathrm{N}_{2}$-Festtemperaturdewar $(77 \mathrm{~K})$ bzw. der Temperaturvariator STT 3 (Hersteller: ZWG der AdV der DDR), der eine Probentemperierung im Bereich von $80 \mathrm{~K}$ bis $550 \mathrm{~K}$ mit einer Genauigkeit von $0,1 \mathrm{~K}$ ermöglicht.

Die Akkumulation und Datenverdichtung der EPR-Spektren (Glättung, Nullinienkorrektur usw.) erfolgte beim ERS 220 im on-line-Betrieb mit dem Kleinrechner KRS 4200 (Hersteller: ROBOTRON, DDR) unter Verwendung der Interface-Einheit IFE 1 (Hersteller: ZWG der AdW der DDR) und Nutzung der Software „ERSON 200“.

Die Digitalisierung der CW-Spektren, die am Spektrometer E 4 aufgenommen wurden, erfolgte mit Hilfe der Off-line-Einheit OFE 1 (Hersteller: ZWG der AdW der DDR). Alle Spektrensimulationen einschließlich des Angleichs von Modellspektren an experimentelle Befunde erfolgten mit Hilfe des Programmsystems "COMPAR“ [12] an der Rechenanlage BESM 6 (ZfR der AdW der DDR).

Die untersuchten Verbindungen wurden wie in [7] beschrieben dargestellt bzw. waren kommerziell erhältlich. Alle Verbindungen und Lösungsmittel wurden durch Destillation, Umkristallisation bzw. Zonenschmelzen gereinigt und anhand der Elementaranalyse sowie der Schmelz- und Siedepunkte überprüft. Die Konzentration der zu den Messungen verwendeten Lösungen betrug $\sim 10^{-3} \mathrm{Mol} / \mathrm{l}$.

\section{Ergebnisse und Diskussion}

\subsection{Optisch angeregte Triplettzustände von Fluorenderivaten}

In Tab. 1 sind die Nullfeldparameter einiger Fluorenderivate und aus Vergleichsgründen die entsprechenden Parameter von Biphenyl, Inden und Phenanthren angegeben.

Typisch für diese Verbindungen in ${ }^{3}\left(\pi, \pi^{*}\right)$-Zuständen sind Nullfeldaufspaltungen im Bereich $\sim 0,1 \mathrm{~cm}^{-1}$. Damit ergeben sich in X-Bandspektren Übergänge, die nach $[13,14]$ in Analogie zu denen des Naphthalins bzw. Fluorens als $z$-, $y$ - und $x$-Über- 
Tab. 1. Nullfeldparameter einiger Fluorenderivate ${ }^{a}$. A) Optich angeregte Triplettzustände. B) Triplettgrundzustände. C) Optisch angeregte Triplettzustände der Produkte während der Bestrahlung.

\begin{tabular}{lllll}
\hline Verbindung & $\begin{array}{l}|D| \\
\left(\mathrm{cm}^{-1}\right)\end{array}$ & $\begin{array}{l}|E| \\
\left(\mathrm{cm}^{-1}\right)\end{array}$ & $\begin{array}{l}\left|D^{*}\right| \\
\left(\mathrm{cm}^{-1}\right)\end{array}$ \\
\hline A & Biphenyl & 0,1114 & 0,0038 & 0,1119 \\
& Fluoren & 0,1075 & 0,006 & 0,1033 \\
& Inden & 0,1010 & 0,0153 & 0,1084 \\
Phenanthren & 0,1039 & 0,0461 & 0,1310 \\
Bifluorenyliden & - & - & 0,0865 \\
9,9'Bifluorenyl & 0,1014 & $\cong 0$ & 0,1013 \\
2-Nitrofluoren & 0,0904 & 0,0132 & 0,0965 \\
2-Aminofluoren & 0,0881 & 0,0139 & 0,0914 \\
2,2-Difluorofluoren & 0,1097 & 0,0013 & 0,1099 \\
9-Bromfluoren & 0,1075 & 0,0032 & 0,1065 \\
9-Aminofluoren & 0,1136 & $\approx 0$ & 0,1067 \\
B & Diphenylcarben & & & \\
& Fluorenyliden & 0,4052 & 0,0194 & \\
2,4,7-Trinitro- & 0,4129 & 0,027 & \\
fluorenyliden & $\cong 0,403$ & & \\
2-Nitrofluorenyliden & 0,4082 & 0,0271 & \\
C & 9-Diazofluoren & 0,1038 & 0,0032 & \\
2-Nitro-9-diazofluoren & 0,0887 & 0,0012 & \\
2,4,7-Trinitro- & $\cong 0,085$ & 0,0011 & \\
9-diazofluoren & & & \\
\hline
\end{tabular}

a Die Nullfeldparameter werden unter Vernachlässigung der Anisotropie von $\tilde{g}$ berechnet. In einigen Fällen kann anhand der unsymmetrischen Lage der Feinstrukturlinien bezüglich $g_{0}$ auf eine schwache Anisotropie des $g$-Tensors geschlossen werden.

b siehe [16]. c siehe z. B. [17].

gänge klassifiziert werden können $(x, y, z$ : Molekülkoordinaten).

Ein Vergleich der Feinstrukturparameter von Naphthalin $\left(|D|=0,0996,|E|=0,0152 \mathrm{~cm}^{-1}\right)$, mit denen von Biphenylderivaten [15] zeigt, daß letztgenannte durch große $|D|$ - und relativ kleine $|E|$ Werte gekennzeichnet sind. Die Unterschiede in den Nullfeldparametern für verschiedene Diphenylderivate sind gering.

Es ist zu erwarten, daß die Substitution des Fluorens in 2-Position einen stärkeren Einfluß auf $\operatorname{die}^{3}\left(\pi, \pi^{*}\right)$-Zustände als die in 9-Position ausübt. Diese Annahme wird durch die Beträge der Nullfeldparameter (Tab. 1) bestätigt. Interessant ist in diesem Zusammenhang die starke Verkleinerung des | $D \mid$-Parameters beim strukturverwandten Inden in bezug auf Fluoren. Im Zusammenhang mit den 2.2' verbrückten Biphenylen wurden die $\left|D^{*}\right|$-Werte ausgewählter phenylsubstituierter Verbindungen bestimmt, von denen einige Strukturelemente analog $1 \mathbf{b}$ aufweisen. Aus Tab. 2 folgt, daß die $\left|D^{*}\right|$-Werte mit Zunahme der Delokalisierungsmöglichkeiten erniedrigt werden. Generell ist ein großer Einfluß der Brückengruppierung " $\mathrm{X}$ “ in $\mathbf{1} \mathrm{c}$ auf die Feinstruk-

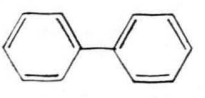

1a

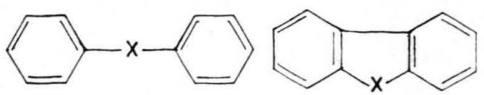

$\underline{16}$ $\underline{1 c}$ tur $\operatorname{der}^{3}\left(\pi, \pi^{*}\right)$-Zustände nicht zu erwarten. Eine Ausnahme bildet Schwefel bzw. Sauerstoff als Brükkenatom.

Bei den Fluorenderivaten tritt eine deutliche Verkleinerung der $|D|$-Werte infolge der Amino- bzw. Nitrosubstitution in 2-Position ein. Die Fluorsubstitution bewirkt ähnlich wie bei den Diphenylderivaten eine Erhöhung der $|D|$-Werte. Analogien sind ebenfalls zwischen den $|E|$-Werten beider Substanzklassen festzustellen. Die Aminosubstitution bewirkt eine Erhöhung, während eine Difluorsubstitution zu einer starken Absenkung des $|E|$-Wertes führt. Sowohl für Diphenyl- als auch für Fluorenderivate wurde ein Zusammenhang zwischen den Nullfeldparametern und Hammett-Konstanten gefunden [15]. Eine lineare Beziehung konnte nicht hergestellt werden.

\subsection{Triplettgrundzustände}

Photochemisch induzierte Reaktionen von 9-Diazofluorenderivaten durchlaufen verschiedene Grundund angeregte Zustände, unter anderem Triplettzustände. Carbenzwischenstufen können in Abhängigkeit von Reaktionspartnern aus verschiedenen elektronischen Zuständen heraus reagieren. Prinzipiell lassen sich EPR-spektroskopisch Aussagen über die Bildung paramagnetischer Zwischenprodukte sowie

Tab. 2. $\left|D^{*}\right|$-Werte einiger Verbindungen des Types $1 \mathbf{b}$ bzw. 1e.

\begin{tabular}{ll}
\hline Verbindung & $\left|D^{*}\right|\left(\mathrm{cm}^{-1}\right)$ \\
\hline Diphenylmethan & $0,1263:(0,1007=|E|)$ \\
1,2-Diphenylethan & $0,1091:(0,0819=|E|)$ \\
2,2'Diphenylpropan & 0,1088 \\
Triphenylmethan & 0,0719 \\
Triphenylcarbinol & 0,1070 \\
Diphenylamin & 0,0899 \\
Triphenylamin & $0,076 \quad(|D|=0,067:|E| \approx 0)$ \\
Carbazol & 0,1006 \\
N-Phenylcarbazol & $0,1057 \quad(|D|=0,1039:|E|=0,0064)$ \\
Dibenzofuran & 0,1139 \\
Dibenzothiophen & 0,1139 \\
Dibenzosulfoxid & 0,1137 \\
Dibenzosulfolan & 0,1137 \\
\hline
\end{tabular}




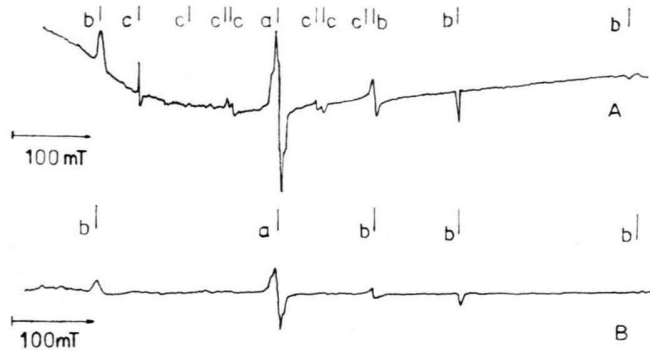

Abb. 2. EPR-Spektrum von bestrahltem 9-Diazofluoren, Lösungsmittel Toluen; $77 \mathrm{~K}$;

A) Im photostationären Zustand (Bestrahlungszeit $20 \mathrm{~min}$ )

a) Dublettgrundzustand ( $g \rightleftharpoons 2$, freies Radikal),

b) Triplettgrundzustand (Fluorenyliden),

c) Optisch angeregte Triplettzustände (s. Tabelle $1 \mathrm{c}$ ).

B) Nach einer Bestrahlungszeit von einer Minute.

über Desaktivierungsprozesse von Triplettzuständen an der Reaktion beteiligter Partner einschließlich Produkte gewinnen. Auf der Basis identifizierter Zwischenprodukte sowie der Kenntnis selektiv besetzter Zustände ist es möglich, Beiträge zur Aufklärung des Reaktionsmechanismus zu liefern (siehe [2]). Im photostationären Zustand wurde bei der Bestrahlung $(\lambda=365 \mathrm{~nm})$ von 9-Diazofluoren in Toluen $(77 \mathrm{~K})$ das in Abb. 2 dargestellte EPRSpektrum erhalten. Es stellt eine Überlagerung der Spektren des Fluorenylidens (Triplettgrundzustand), der optisch angeregten Tripletts und radikalischer Spezies dar. Die Annäherung an den photostationären Zustand erfolgt dabei entsprechend den Geschwindigkeitskonstanten für die Bildung des Carbens und der Produkte (s. Schema [2]). Bei Mes-
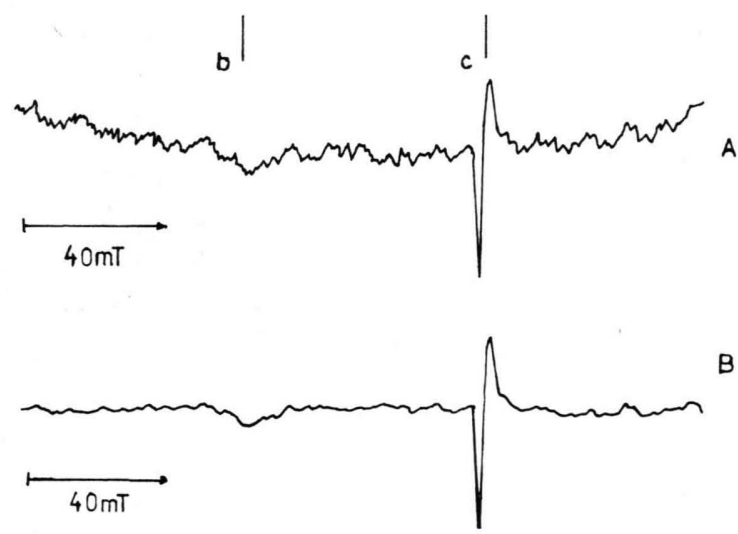

Abb. 3. EPR-Spektrum von bestrahltem 2,4,7-Trinitro-9. Diazofluoren im Bereich von 60 bis $200 \mathrm{mT}$, Lösungsmittel Toluen; $77 \mathrm{~K}$ :

A) Nach zehnfacher Akkumulation,

B) Wie A, zusätzlich Basislinienkorrektur und Glättung. sungen im X-Band $\left(h v \approx 0,3 \mathrm{~cm}^{-1}\right)$ gilt $|D| \gtrless h v$ für die optisch angeregten bzw. Triplettgrundzustände (s. 3.1.). Zum Nachweis der entsprechenden Triplettzustände des 2.4.7-Trinitro-9-diazofluorens mußten infolge der geringen stationären Triplettkonzentration die Spektren akkumuliert werden (s. Abbildung 3).

Die Beteiligung von Produkten an den in Abb. $2 \mathrm{~A}$ dargestellten Spektren wird im Vergleich zu Abb. 2 B deutlich. Hier wurde das gleiche System nur $60 \mathrm{~s}$ unter gleichen Bedingungen bestrahlt. Ein photostationärer Zustand stellt sich in Abhängigkeit von der Konzentration der Lösung und der Zahl der absorbierten Photonen nach etwa 20 min ein.

Zum Verständnis der vorliegenden Spektren, als Zuordnungshilfe sowie zur Bestimmung der Nullfeldparameter wurden Simulationen durchgeführt (vgl [12]). Der Berechnung wurde eine chaotische Verteilung der Moleküle im Toluenglas zugrunde gelegt. Wiederholungsexperimente bei veränderter Orientierung in bezug auf $\boldsymbol{B}_{0}$ zeigten, daß diese Annahme auch unter den Bedingungen photochemischer Reaktionen näherungsweise gerechtfertigt ist.

Für die Zuordnung der Übergänge in glasartigen Proben ist die Kenntnis der Winkelabhängigkeit der Resonanzwerte der magnetischen Induktion $\boldsymbol{B}$ und der entsprechenden Übergangswahrscheinlichkeiten von Bedeutung. Anhand von Abb. 4 und 5 wird der Einfluß der Nullfeldaufspaltung auf die genannten Parameter deutlich. Damit läßt sich der völlig verschiedene Spektrenhabitus von optisch angeregten und Triplettgrundzuständen der hier untersuchten

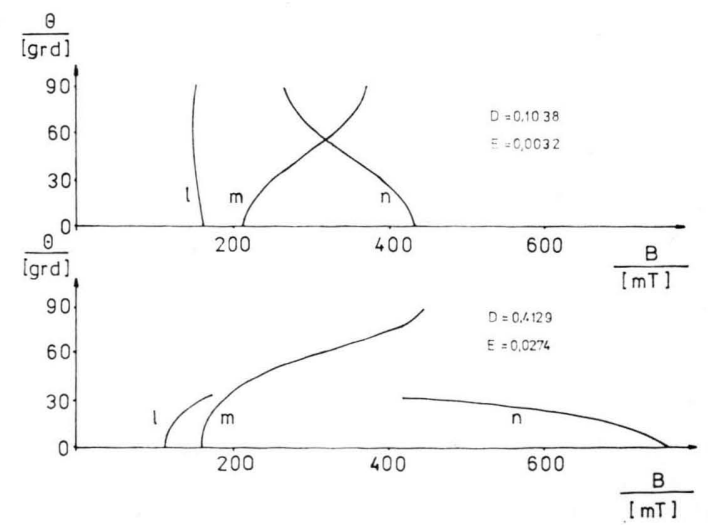

Abb. 4. Abhängigkeit der Resonanzwerte von $\boldsymbol{B}$ der Feinstrukturübergänge $l, m, n$ von der Orientierung der Moleküle $(\theta, \varphi)$ in bezug auf die äußere magnetische Induktion $\boldsymbol{B}_{0}$ bei $\varphi=0^{\circ}$. 


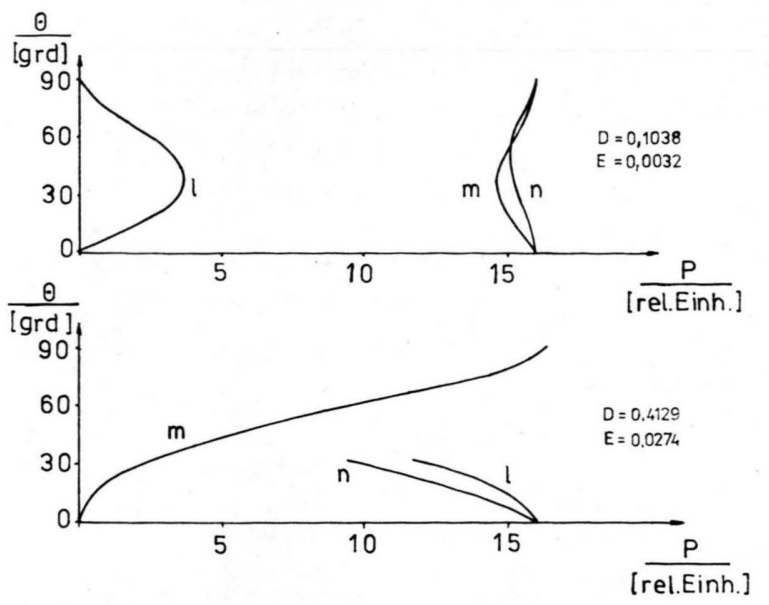

Abb. 5. Abhängigkeit der relativen Utbergangswahrscheinlichkeit $P$ von der Orientierung der Moleküle $(\theta, \varphi)$ in bezug auf $\boldsymbol{B}_{0}$ bei $\varphi=0^{\circ}$.
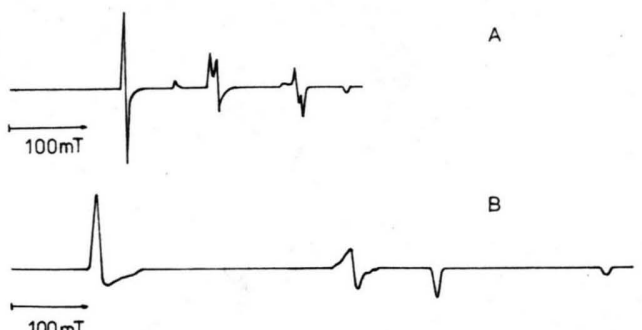

Abb. 6. Simulierte EPR-Spektren (Programm COMPAR [12] für das System 9-Diazofluoren/Toluen).

A) Fluorenyliden; Nullfeldparameter entsprechend Tabelle $1 \mathrm{~B}$.

B) Optisch angeregter Triplettzustand; Nullfeldparameter entsprechend Tabelle $1 \mathrm{C}$.

organischen Moleküle ohne weitere Annahmen erklären (vgl. Abbildung 6).

\subsection{Radikale}

Radikale und Ionenradikale von Fluorenderivaten in stationären Konzentrationen waren bereits oft Gegenstand von EPR-Untersuchungen, die zusammen mit Modellberechnungen zu Vorstellungen über Spindichteverteilungen im Grundzustand führten [1 - 4]. Bei den hier vorgestellten Untersuchungen konnten bei Bestrahlung der 9-Diazofluorene in festen Matrizen radikalische Spezies im CW-Betrieb registriert werden. Hyperfeinstrukturen konnten dabei nicht eindeutig aufgelöst werden. Bei Temperaturerhöhung oberhalb des Glaspunktes bzw. beim Erweichen der festen Lösungsmittelmatrix setzte die unmittelbare
Rekombination der Radikale ein. Deshalb wurden entsprechend Abb. 1 zeitaufgelöste Untersuchungen im Temperaturbereich von 150 bis $300 \mathrm{~K}$ durchgeführt. In diesem Temperaturbereich konnten keine Feinstrukturen von Triplettzuständen nachgewiesen werden.

\subsubsection{Folgereaktionen der Fluorenyl- idine in Ethanol}

Bei der Photolyse von 9-Diazofluoren in Gegenwart von Ethanol ist nach der optischen Anregung in Lösung ab $170 \mathrm{~K}$ im Bereich $g \approx 2$ ein Dublett $(a \approx 0,6 \mathrm{mT})$ zu beobachten, das dem Fluorenylradikal zugeordnet werden kann (s. a. [2]). Es kann durch H-Abstraktion des Fluorenylidens aus dem Lösungsmittel gebildet werden, das eine typische Triplett-Carben-Reaktion darstellt. Demgegenüber wird auch die Bildung des Fluorenylradikals aus dem Singulett-Zustand des Carbens diskutiert [18]. Durch Rekombinationsreaktionen entstehen stabile diamagnetische Endprodukte (siehe [2]). Ein Vergleich des Intensitäts-Zeitverhaltens des Signals des Fluorenylradikals mit dem des Benzophenon-Ketylradikals $\left(k=10^{6} \mathrm{~s}^{-1}(\mathrm{Mol} / \mathrm{l})^{-1}\right)$ unter vergleichbaren experimentellen Bedingungen ergibt, da $\beta$ die Geschwindigkeit der Folgereaktion des Systems 9. Diazofluoren/Ethanol im Bereich $k=10^{8} \mathrm{~s}^{-1}(\mathrm{Mol} /$ 1) ${ }^{-1}$ liegt.

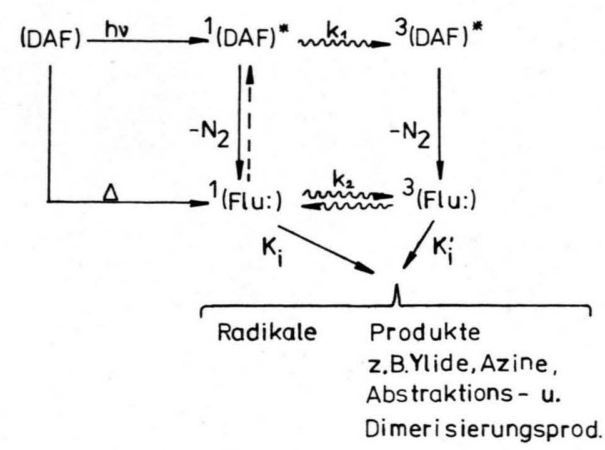

3.3.2. Folgereaktionen der Fluorenylidene in Pyridin

In Gegenwart von Pyridin werden in Lösung $(T=230 \mathrm{~K})$ neben Fluorenylradikalen tiefblau gefärbte, sehr reaktive Spezies (diamagnetische) erhalten. Sie wurden aufgrund ihres charakteristischen UV/VIS-spektroskopischen Absorptionsverhaltens 
$\left(\lambda_{\max }: 610 \mathrm{~nm}\right.$ (Pyridin) ) dem 1-(9-Fluorenyl-) pyridinium-Ylid $\left(\lambda_{\max }: 597 \mathrm{~nm}, \varepsilon_{\max }: 21400 \mathrm{~mol}^{-1}\right.$ $\mathrm{cm}^{-1}(\mathrm{DMF})$ zugeordnet [6].

Der Einfluß geeigneter Triplettsensibilisatoren auf die photochemisch induzierte Betain-Bildung sowie

[1] F. Gerson, B. Kowert u. B. M. Peake, J. Amer. Chem. Soc. 96, 118 (1974).

[2] F. A. Neugebauer u. W. R. Groh, Tetrahedron Lett. $1973,1005$.

[3] D. K. Gupta, C. P. Poole, Jr. u. H. A. Farach, Lett. Nuovo Cim. Ser. 2, 2, 20 (1971).

[4] K. Möbius u. M. Plato, Z. Naturforsch. 22a, 929 (1967).

[5] C. A. Hutchison, Jr., Pure Appl. Chem. 27, 327 (1971).

[6] R. Stößer, J.-U. Thurner, R. Dehmlow u. G. Tomaschewski, Z. Chem. 19, 346 (1979).

[7] J.-U. Thurner, R. Stößer u. G. Tomaschewski, in Vorbereitung.

[8] H. Dürr, Topics Curr. Chem. 55, 87 (1975).

[9] V. I. Savin, Ž. vses. Chim. obšč. 24, 454 (1979).

[10] V. Zimmermann, H. C. Wolf u. M. Schwoerer, Chem. Phys. Lett. 31, 401, 406 (1975).

[11] R. Stößer, J.-U. Thurner u. G. Tomaschewski, Schultagung ,Moderne Methoden der Hochfrequenzspek- der Vergleich mit entsprechenden thermischen Reaktionen ist Gegenstand weiterer Untersuchungen (s. a. [7]) und kann weitere Beiträge zur Klärung der Rolle der Triplettzustände im Reaktionsmechanismus entsprechend [2] erbringen.

troskopie“" Physikalische Gesellschaft der DDR, Reinhardsbrunn 1981, S. 265.

[12] U. Ewert, Dissertation A, Humboldt-Universität zu Berlin 1979.

[13] R. Stößer, J.-U. Thurner, T. Hanke u. G. Sarodnick, J. prakt. Chem. im Druck.

[14] M. S. de Groot u. J. H. van der Waals, Mol. Phys. 3, $190(1960)$.

[15] T. Hanke, Diplomarbeit, Humboldt-Universität zu Berlin, Sektion Chemie, 1979.

[16] J. Mispelter, J. P. Grivet, M. Baiwir u. J. M. Lhote, Mol. Phys. 24, 205 (1972).

[17] J. K. S. Wan. in "Advances in Photochemistry" ed. J. N. Pitts, G. S. Hammond u. K. Gollnick, WileyInterscience, John Wiley u. Sons, Inc. New York 1974, Bd. 9, S. $1 \mathrm{ff}$.

[18] P. C. Wong, D. Griller u. J. C. Scaino, J. Amer. Chem. Soc. 103, 5934 (1981). 